\title{
Dynamic representation of the fundamental diagram via Bayesian networks for estimating traffic flows from probe vehicle data
}

\author{
Thorsten Neumann ${ }^{1}$ and Philipp L. Böhnke ${ }^{2}$ and Louis C. Touko Tcheumadjeu ${ }^{3}$
}

\begin{abstract}
Area-wide measurements of traffic flow are usually not possible with today's common sensor technologies. However, such information is essential for (urban) traffic planning and control. Hence, in order to support traffic managers, this paper analyses an approach for deriving traffic flows from probe vehicle speeds, which are potentially available with a wide spatial coverage. The idea is to apply the speed-flow function as known from macroscopic traffic flow theory. In this context, a stochastic representation of the fundamental diagram via Bayesian networks is proposed which also considers the temporal dependencies and transitions between the appearing traffic states. The paper describes the relevant theoretical concepts in comparison to the traditional approach of fitting deterministic curves to empirical speed-flow relations. Moreover, it analyses the findings of an extensive validation in context of traffic flow estimation via probe vehicle data using real traffic measurements provided by about 600 local detectors and about 4,300 taxi probes in Berlin, Germany.
\end{abstract}

\section{INTRODUCTION}

The discussion about the detailed relationship between the three traffic state variables flow, density and speed has a long history (cf. [1]) and is still ongoing. Starting from the pioneering works of Bruce D. Greenshields in the 1930s (see [2]), many attempts have been made to find the optimal fit between mathematical models and empirical findings (cf. [3], [4], [5]). A quite flexible but still deterministic model, for instance, was proposed by Michel van Aerde in 1995 (see [6]) which is based on a microscopic assumption about vehicle headways. Recent research (cf. [7]) more and more tries to explicitly model the stochastic aspects of the fundamental diagram. Moreover, there are promising approaches and applications in context of exploring networkscaled relations between vehicle density and space-mean flow - called macroscopic fundamental diagram (cf. [8], [9]). The present contribution, however, concentrates on link-wise flows and speeds as common theories do.

During the last decades, a large number of measurement techniques has been developed for observing the relevant traffic state variables from an empirical perspective. In this context, traffic flow and time-mean speed (even more than density) play a major role as they can easily be measured locally and are an important input to current tools of traffic planning and control. Moreover, space-mean speeds as appearing in the well-known fundamental equation of traffic flow can be computed directly from local speed

All authors are with German Aerospace Center (DLR), Institute of Transportation Systems, Rutherfordstr. 2, 12489 Berlin, Germany

${ }^{1}$ Corresponding author: Thorsten. Neumannedlr.de

${ }^{2}$ Philipp.Boehnke@dlr.de

${ }^{3}$ Louis. ToukoTcheumadjeu@dlr.de measurements under certain assumptions by applying the harmonic mean (cf. [3]). In contrast to that, today's methods do not allow for area-wide measurements of traffic flow, for instance, due to the fact that common sensors like loop detectors always refer to fixed locations of the road network.

On the other hand, more recent sensor approaches such as probe vehicle data (PVD) or similar means (cf. [10], [11]) have a wide spatial coverage regarding their measurements but do not provide information about traffic state variables such as flow or density. Consequently, it is straight-forward to ask whether it is possible to combine the advantages of local detectors (e.g. direct measurements of traffic flow) with the ability of probe vehicle systems to provide area-wide traffic measurements. Examples for such data fusion approaches can be found in [12], [13], [14], [15], for instance. Moreover, a quite simple idea would be making use of the speed-flow relation as known from macroscopic traffic flow theory in order to estimate traffic flows based on measured speeds from PVD.

Reference [16] describes the results of a concrete implementation (mostly for urban traffic) using calibrated speedflow functions of the Van Aerde model as in [6] based on hourly averaged data. It is shown that the approach is more or less applicable, in principle. However, the deterministic modelling of the speed-flow relation turns out to be too restrictive in such a way that it does not adequately capture the variations of observed speeds given the same traffic flow. Because of that, the present paper proposes a more detailed representation of the fundamental diagram (i.e. speed and flow) based on stochastic Bayesian networks (see Section II) which also takes account of the dynamic transitions between the macroscopic traffic states over time (cf. [17]). Section III then describes the application of this representation for estimating traffic flows from PVD. The paper closes with a short conclusion (see Section IV) summarizing the main results and discussing some aspects regarding future improvements.

\section{MODELLING SPEED-FLOW RELATIONS}

Fig. 1 shows a sample data set of the speed-flow relation based on measurements of a local detector. As can be seen, its general structure is recovered by the approximative function quite well which was obtained by numerically fitting the parameters of the Van Aerde model [6] for the given (hourly) data. However, the observed variance of speed given the flow (and vice versa) is not represented.

Consequently, it might be more reasonable in modelling to think of speed and flow as time dependent random variables where $Q(t)$ is the flow and $V(t)$ the speed at time $t$. The 


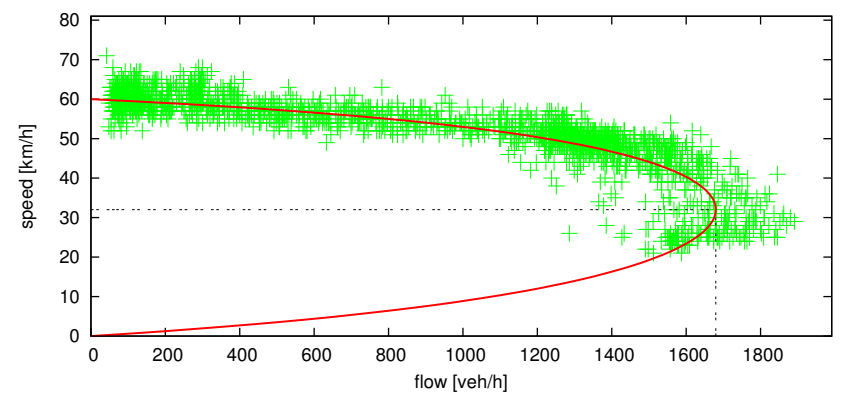

Fig. 1. Example of a speed-flow relation.

stochastic relation between $Q(t)$ and $V(t)$ is then given by the conditional probability distribution $\mathbb{P}(V(t) \mid Q(t))$. Moreover, the opposite relation can be computed via Bayes' Theorem (cf. [18])

$$
\mathbb{P}(Q(t) \mid V(t))=\frac{\mathbb{P}(V(t) \mid Q(t)) \cdot \mathbb{P}(Q(t))}{\mathbb{P}(V(t))}
$$

where $\mathbb{P}(Q(t))$ is the a priori distribution for the observed flows and

$$
\mathbb{P}(V(t))=\sum_{q} \mathbb{P}(V(t) \mid Q(t)=q) \cdot \mathbb{P}(Q(t)=q) .
$$

Fig. 2 shows a graphical model of the connection between $Q(t)$ and $V(t)$. In fact, this model is a trivial example of so-called Bayesian networks (cf. [18], [19]) which efficiently describe stochastic (in-)dependencies for given sets of random variables. In this context, each random variable is represented by a node of a directed acyclic graph. Moreover, edges describe the direct stochastic (sometimes also causal) influence between neighboring nodes.

Then, depending on the graph structure, there are three different types of nodes: A child node is such a one with at least one directed edge pointing at it. Conversely, parent nodes have at least one edge pointing away from it at one of its child nodes. Finally, nodes which do not have any parents are called root nodes. Of course, root or child nodes can also be parent nodes at the same time. But, only root nodes are never the child of any other node per definition.

Based on that, a Bayesian network is completely specified by its graph structure together with the a priori distributions $\mathbb{P}(X)$ of all root nodes $X$ and the conditional probabilities $\mathbb{P}(Y \mid \mathrm{Pa}(Y))$ for each other node $Y$ given its parents $\mathrm{Pa}(Y)$. Regarding Fig. 2, that means the model is fully defined by the probability distributions $\mathbb{P}(Q(t))$ and $\mathbb{P}(V(t) \mid Q(t))$ as already discussed above.

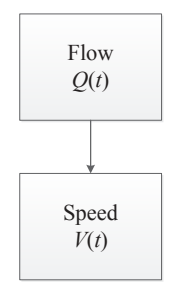

Fig. 2. A trivial Bayesian network.

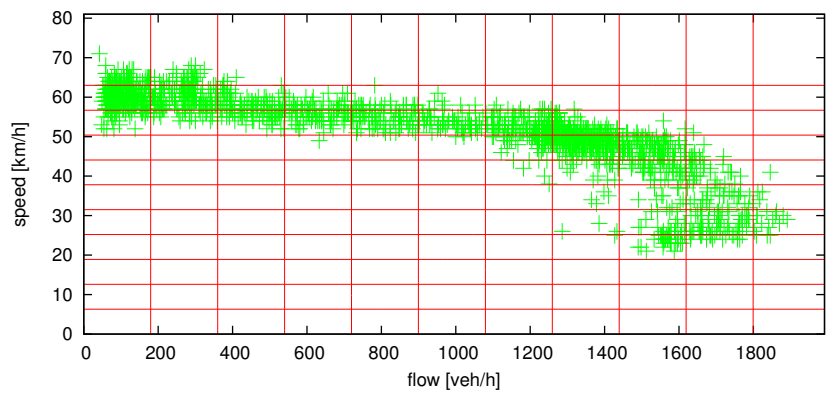

Fig. 3. Discretization of the speed-flow diagram.

Clearly, both distributions are directly obtained from the measurements as depicted in Fig. 1. In this context, common software tools for Bayesian networks (cf. [20]) use probability tables for coding the (conditional) distributions of each node and thus are restricted to discrete random variables for technical reasons. Consequently, the axes of the speed-flow diagram in Fig. 1 need to be discretized in the following. Using equidistant partitions for both directions (cf. Fig. 3), all relevant probabilities can be assigned then by simply counting the data points in each box and normalizing the results. Of course, other kinds of discretization are possible as well, including those which are more fine-grained in regions of the fundamental diagram where data points cluster. For simplicity, however, the present paper sticks to the equidistant variant as in Fig. 3.

Even though stochastically, the speed-flow relation has been modelled statically so far. That means dynamic transitions between macroscopic traffic states over time are not represented in the model. In this way, for instance, the static model does not distinguish between short-term peak traffic and long-lasting jams resulting in the same average speed at time $t$. On the other hand, one can imagine that flows may differ significantly in such a case. Needless to say, the traditional approaches for fitting the fundamental diagram (cf. [2], [4], [5], [6]) have the same restrictions. Analyses as in [17], however, showed that there are distinct transitions between macroscopic traffic states which are more likely than others. That means, the recent history of traffic states has an important dynamic impact on current flows which needs to be considered.

Consequently, the model from Fig. 2 is extended below in such a way that it reproduces these dynamics. Interestingly, it is very simple to do that for the Bayesian network approach (in contrast to the traditional fitting approaches). So, there is no reason to stick to the static model. The only thing to do is replicating the trivial network from Fig. 2 for a number of consecutive time slices in the past and to connect them via some additional edges. Under the assumption that the random flow $Q(t-1)$ at time $t-1$ directly influences the flow $Q(t)$ at time $t$ in a stochastic way, for instance, these additional edges would be between the nodes $Q(t-1)$ and $Q(t)$ for all $t$.

Of course, this dynamic extension does not necessarily mean that the model will be fed in real-time with online 

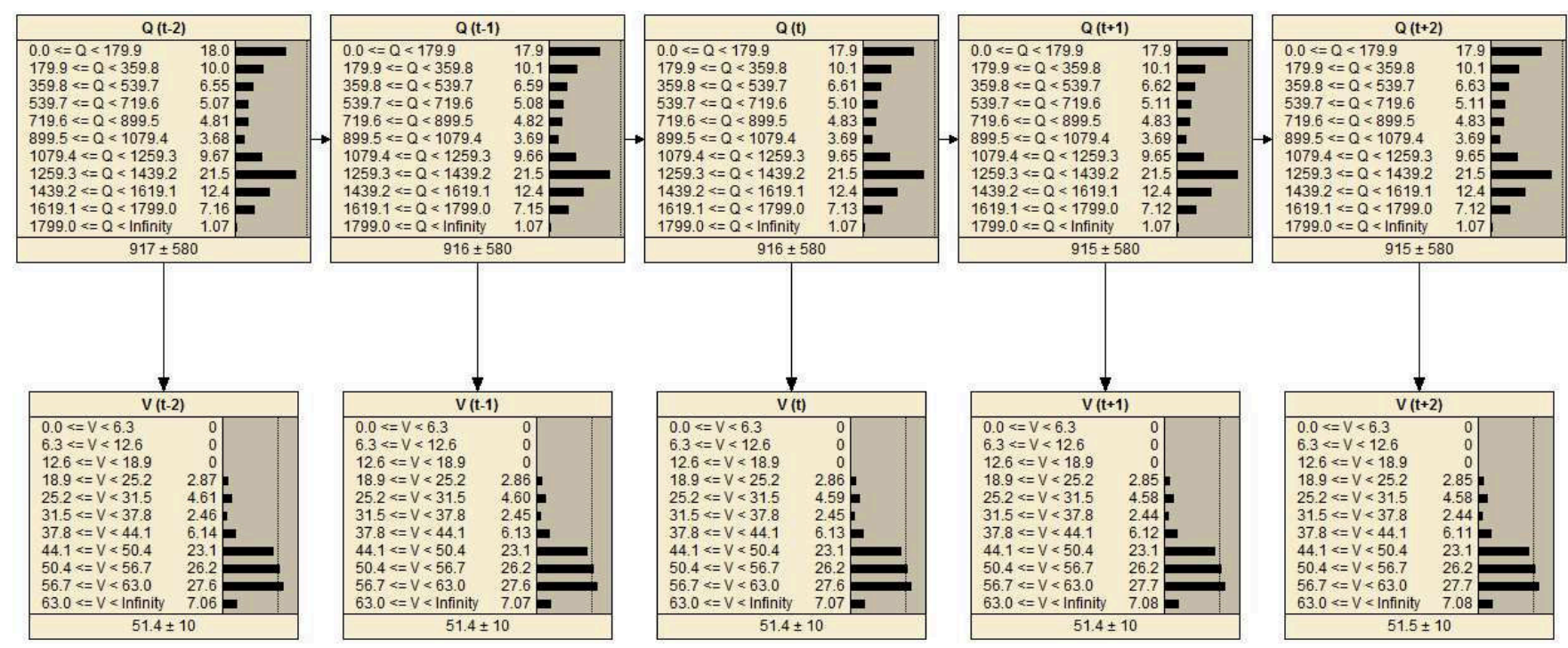

Fig. 4. Dynamic representation of the speed-flow relation.

data during flow estimation (although this would be possible, too). In fact, even the most relevant time slice $t$ will typically be in the past so that it will be standard to use the model mostly offline with historical data in context of less timecritical transport planning applications. The main benefit in this context is that aera-wide estimates of traffic flow, which cannot be obtained by conventional local detectors, are provided rather than real-time traffic states.

Fig. 4 shows the Bayesian network based on previous data in case of two additional backward $(t-2$ and $t-1)$ and two forward time slices $(t+1$ and $t+2)$ as it was implemented using the software tool Netica 4.16 (see [20]). The duration of each time slice is 1 hour so that the time horizon is able to completely cover a typical peak period, for instance. Needless to say, the time slices $t+1$ and $t+2$ will be ignored in the above shortly addressed online case, of course, as they were located in the future then.

Now, a full specification of the model requires knowledge about all of the following probability distributions:

- $\mathbb{P}(Q(t-2))$,

- $\mathbb{P}(V(t+i) \mid Q(t+i))$ for all $i=-2,-1,0,1,2$,

- $\mathbb{P}(Q(t+i) \mid Q(t+i-1))$ for all $i=-1,0,1,2$.

However, claiming time homogeneity (i.e. that all above probabilities are constant over time), this number reduces to three probability distributions, two of which already known from the model of Fig. 2. Namely,

$$
\begin{aligned}
\mathbb{P}(Q(t-2)) & =\mathbb{P}(Q(t)), \\
\mathbb{P}(V(t+i) \mid Q(t+i)) & =\mathbb{P}(V(t) \mid Q(t)), \\
\mathbb{P}(Q(t+i) \mid Q(t+i-1)) & =\mathbb{P}(Q(t) \mid Q(t-1))
\end{aligned}
$$

for all $i$. Moreover, the new distribution from (5) can directly be obtained from local flow measurements as the others, too, without any problem. The model in Fig. 4, for instance, is completely calibrated based on the data set from Fig. 3 . Then, Fig. 4 depicts the resulting marginal probabilities for all possible (discrete) node states in the "stationary" case including the distribution from (3) where no further information about current speeds and flows is given. In addition, Fig. 5 and Fig. 6 show the discretized cumulative distribution functions belonging to (4) and (5).

\section{TRAFFIC FLOW ESTIMATION}

Obviously, the marginal probabilities from Fig. 4 are not very helpful regarding traffic flow estimation because they just represent a whole day average with a constant mean value over time which is more or less the daily traffic volume devided by 24 hours. In order to obtain true hourly estimates, speed measurements are needed as so-called evidence (cf.

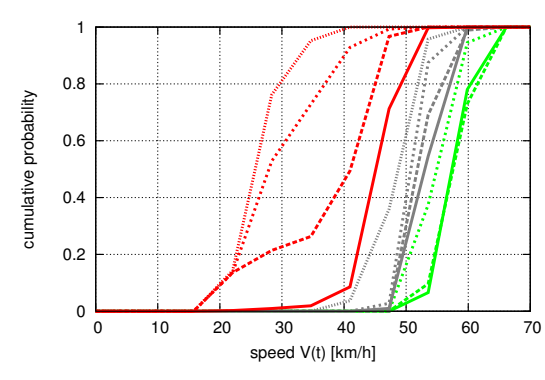

Fig. 5. Cumulative distribution functions of $V(t)$ given $Q(t)$.
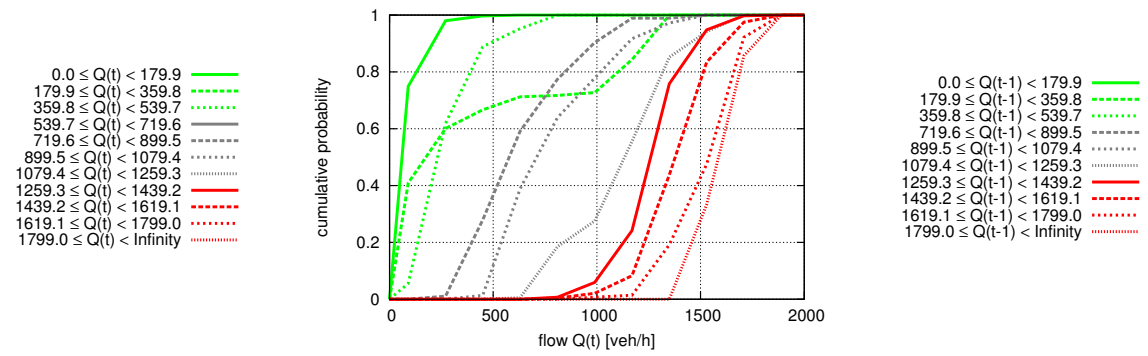

Fig. 6. Cumulative distribution functions of $Q(t)$ given $Q(t-1)$. 

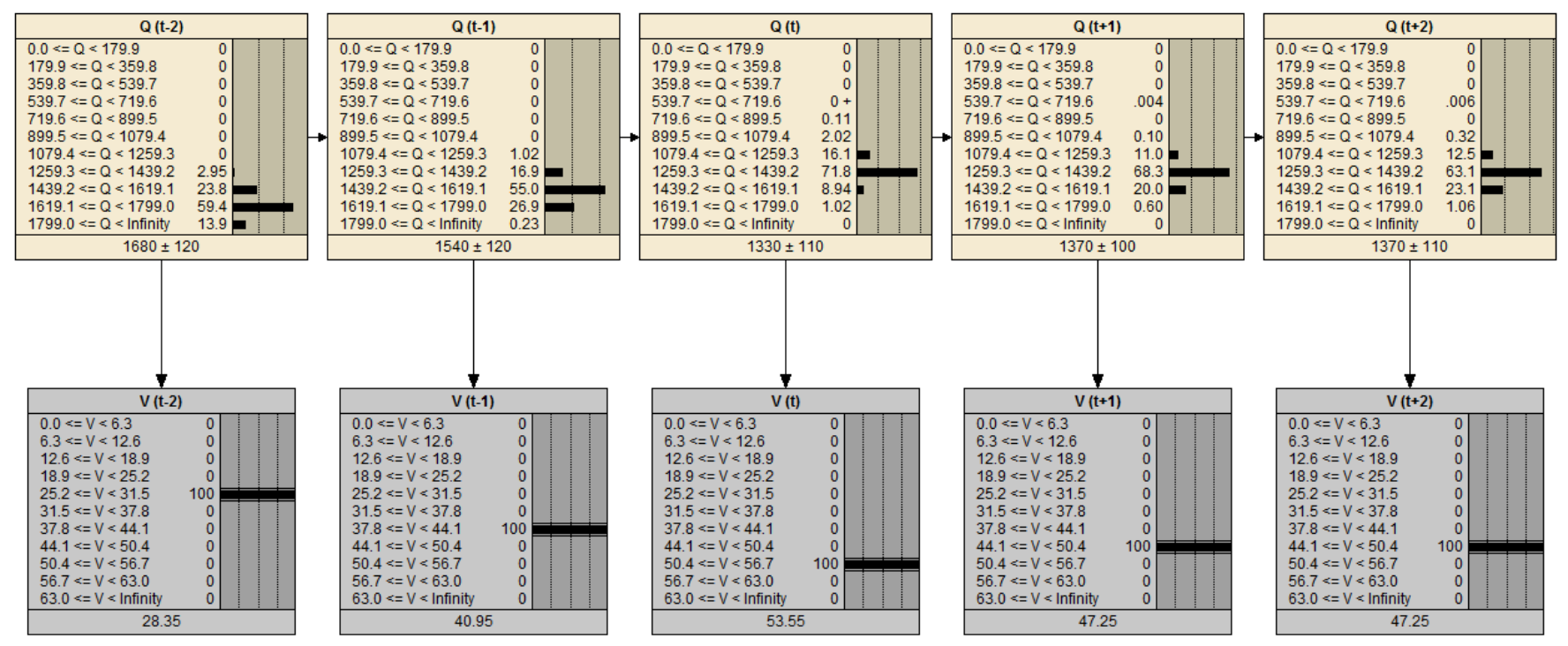

Fig. 7. Traffic flow estimation via speed evidence.

[18], [19]). That means, once the values of some (or all) speed nodes in the Bayesian network of Fig. 4 are fixed, the model is able to recompute the probabilities of all other nodes given this additional information via inference. Fig. 7 shows an example based on some virtual speed measurements representing the relaxation of traffic after a peak hour.

As can be seen, the estimated mean traffic flow decreases from $1,680 \mathrm{veh} / \mathrm{h}$ to $1,330 \mathrm{veh} / \mathrm{h}$ at time $t$ and then stabilizes around $1,370 \mathrm{veh} / \mathrm{h}$ as the discretized speeds do as well at $47.25 \mathrm{~km} / \mathrm{h}$ on average. Moreover, also the (conditional) probability distributions of the nodes $Q(t-2), \ldots, Q(t+2)$ are shifting dynamically in a reasonable way. In contrast to that, the static model from Fig. 2, which does not incorporate the temporal dynamics of the traffic states, would estimate an average flow of just $945 \mathrm{veh} / \mathrm{h}$ at time $t$ based on the same speed measurements as in Fig. 7. Needless to say, applying the more realistic dynamic model from Fig. 4 is the better choice.

\section{A. Approach}

The above example shows that the Bayesian network model is able to (qualitatively) provide suitable estimates of traffic flow given some speed measurements. Needless to say, the calibration of the model strongly depends on factors such as road type, speed limit, number of lanes and possibly others. For this reason, a number of road classes was defined in the following based on these criteria where each road class has its own calibrated Bayesian network. In this context, the parameters of the models were obtained by mapping the speed and flow measurements of about 40 local sensors to their related road classes and then evaluating the discretized data sets according to Section II. Finally, there are calibrated models for all major roads of the considered street network (even those without any local detector) since each of these roads, of course, belong to one of the defined road classes.

The area-wide speed measurements, which are needed as evidence, then come from 3 months of typical probe vehicle data with sampling intervals of about $30 \mathrm{~s}$. That means travel times $\Delta t$ for each road section are derived via common PVD algorithms (cf. [10]) first which yield so-called travel speeds $v_{\mathrm{PVD}}:=L / \Delta t$ as their inverse where $L$ is the driven distance between the corresponding PVD messages. Based on that, typical historical daily curves of speed are derived on an hourly basis for every road section and for each weekday class (i.e. single day, tuesday to thursday, monday to sunday). Again, this shows that the whole approach strongly focuses on offline applications rather than realtime traffic state estimation although feeding the model with online PVD was possible, too. Finally, smoothing of the discussed daily curves is done via Lomb filtering as described in [21] whenever feasible.

As is well-known, travel speeds may significantly differ from local speeds which in particular holds for urban traffic. Unfortunately, the exact relationship between both types of speed is rather complex under real conditions and thus cannot be considered here in detail. Nonetheless, transforming $v_{\mathrm{PVD}}$ is necessary to make the speed axes of the calibrated Bayesian network models (cf. Fig. 3) and probe vehicle speeds more comparable. In particular, the free-flow speed needs to be adapted for each single road section in order to avoid differences of at least the basic speed level (e.g. because of varying speed limits on different links or divergent free-flow speeds in general). For this purpose, even if it is just part-way correct, the proposed approach applies the simple transformation

$$
v_{\mathrm{PVD}}^{\prime}:=v_{\mathrm{PVD}} \cdot \frac{v_{\mathrm{Bayes}}^{(.95)}}{v_{\mathrm{PVD}, 0}}
$$

instead of directly plugging in $v_{\mathrm{PVD}}$ as evidence values during traffic flow estimation. Here, $v_{\mathrm{PVD}, 0}$ is the free-flow speed from PVD, and $v_{\text {Bayes }}^{(.95)}$ is the supposed free-flow speed in the corresponding Bayesian network. Precisely, $v_{\mathrm{PVD}, 0}$ is the link-specific average travel speed between $10 \mathrm{pm}$ and 


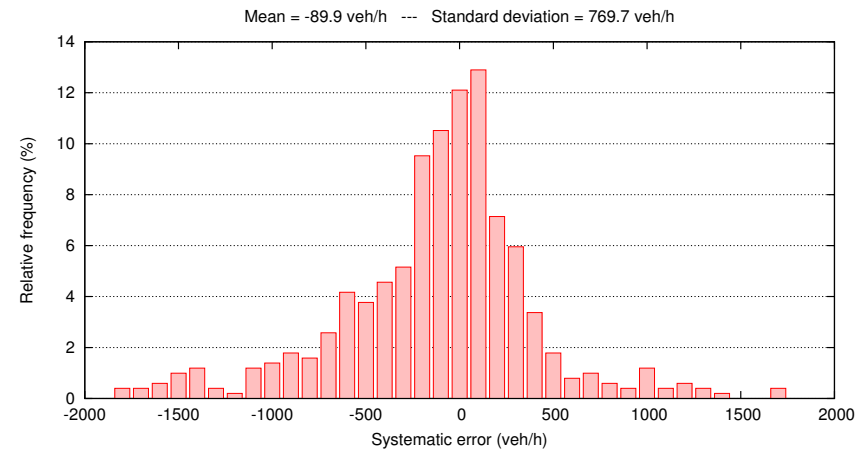

Fig. 8. Aggregated results (tuesday to thursday).

4am (assuming that there is no congestion at night) for the considered road section which is directly derived from the data. Moreover, $v_{\text {Bayes }}^{(.95)}$ is the $95 \%$-percentile of all local speed measurements used for calibrating the model of the related road class.

\section{B. Results}

Fig. 8 shows the aggregated results of an extensive validation using PVD from about 4,300 taxis in Berlin, Germany. In this context, the calibrated Bayesian networks (cf. Fig. 4) were applied for estimating the traffic flows of the central time slice $t$ which means that speed evidences for backward and forward time slices were put into use. Needless to say again, this is only possible in case of the "offline" evaluation based on historical data. During the "online" application, speed measurements will be available for current and backward but not for future time slices, of course. Finally, the estimated flows were compared to the true ones as provided by local detectors for about 600 road links (including those 40 used for calibration) covering all relevant road classes with regard to the "typical" working days (tuesday to thursday).

As can be seen, there is a small systematic bias of about $-89.9 \mathrm{veh} / \mathrm{h}$ in terms of underestimating the true traffic flows by PVD. Moreover, the mean absolute error sums up to $481.6 \mathrm{veh} / \mathrm{h}$. The standard deviation of the error differences is computed as $769.7 \mathrm{veh} / \mathrm{h}$. Clearly, these results are not really satisfying although, for instance, they are better than those from [16] which based on a static and deterministic representation of the fundamental diagram instead of the proposed Bayesian network approach.

In this context, Fig. 9 depicts an example where the estimation works quite well. In particular, notice that the plotted flow data come from a link which does not belong to those 40 used for calibration in this case. The upper diagram shows the true and estimated daily curve of traffic flow while the second one displays the corresponding probe vehicle speeds as well as the average number of PVD messages per hour (cover) which in some way is a quality indicator for the derived speeds.

There is no doubt that a sufficiently good coverage by PVD is essential for reasonable results. Fig. 10 gives an example where low "cover" values are responsible for the unrealistic
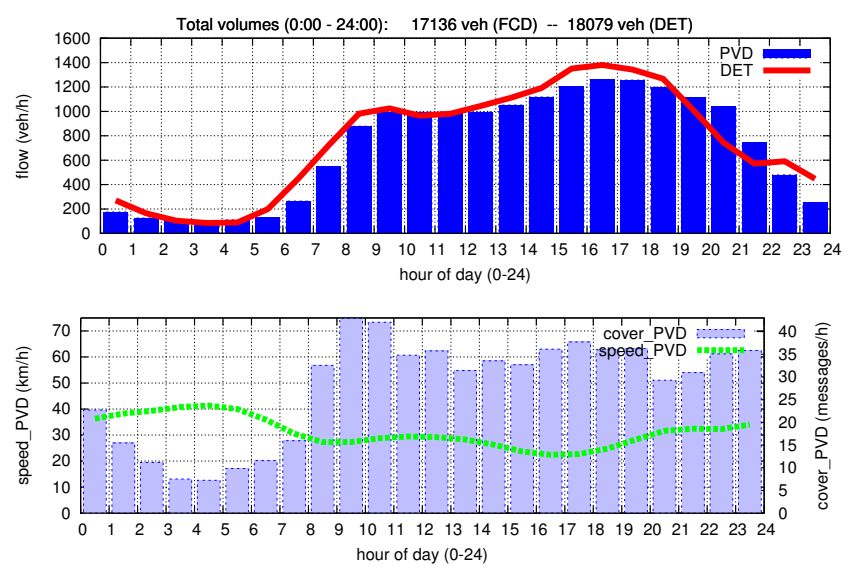

Fig. 9. Exemplary daily curve of estimated flow.
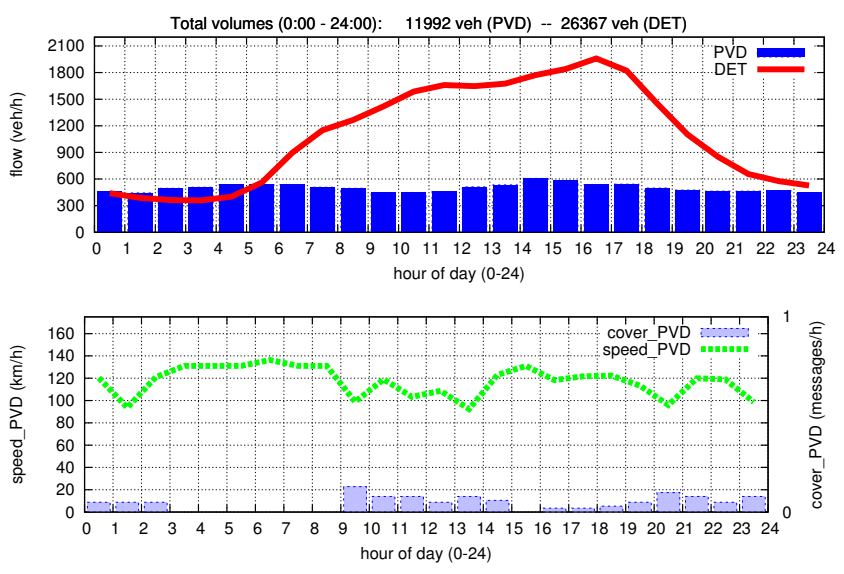

Fig. 10. Negative example of estimated flows.

and noisy speed curve in the lower plot (i.e., notice that the current PVD processor assumes free-flow speed on links where no data are available). Consequently, the estimated traffic flows show a bad quality, too.

However, this is not the only explanation why the estimates sometimes do not reproduce the true flows adequately. The problem is that the speed-flow relation as in Fig. 1 often has a very flat branch in case of free-flow and undersaturation. That means variations of flow do not necessarily induce significant deviations of the corresponding speeds. Obviously, freeways and other roads with high speed limits and large capacities are affected especially hard (cf. [16]).

Finally, the applied road classification scheme (see Section III-A) makes some important potential for estimation errors. Because, using an inappropriate Bayesian network model will of course produce wrong results. Needless to say, this especially holds when roads with significantly different capacities or speed limits are mixed up within a given road class. Unfortunately, optimizing the currently used road classification scheme is a difficult task which cannot be part of this paper.

In order to rate the influence of flat speed-flow relations, 


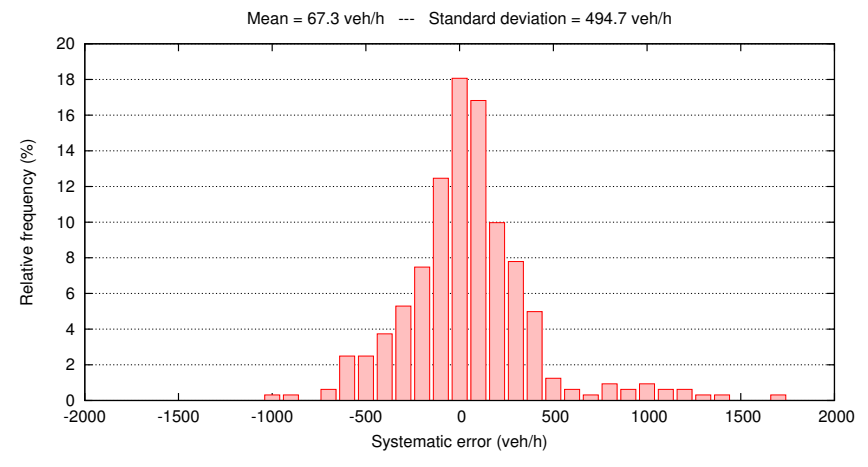

Fig. 11. Aggregated results (without freeways).

however, a second analysis without freeways and similar road classes were done for the same days as before. Fig. 11 shows the aggregated results. As expected, they are more accurate than those from Fig. 8 with a slight tendency to overestimate the true traffic flows now. That is, the systematic bias is found to be $67.3 \mathrm{veh} / \mathrm{h}$. The mean absolute error sums up to $297.3 \mathrm{veh} / \mathrm{h}$, and the standard deviation of the error goes down to $494.7 \mathrm{veh} / \mathrm{h}$.

\section{CONCLUSIONS}

Effective traffic planning and control based on conventional and modern ITS technologies is not possible without reliable traffic information. In particular, there is a significant number of tasks such as traffic signal planning or infrastructure decisions which essentially rely on comprehensive data about traffic flow and demand. For that reason, this paper proposed a stochastic approach based on PVD (and mainly focussing on offline applications) in order to generate traffic flow estimates with a wide spatial coverage since area-wide direct flow measurements are not possible with common local sensors or other today's means.

It principally turned out that the dynamic representation of the fundamental diagram using Bayesian networks is capable of providing reasonable flow results via inference. However, there are several factors negatively influencing the quality. Therefore, future research should try to optimize the implemented road classification scheme in order to create more homogeneous road classes regarding their relevant traffic flow characteristics. In addition, the focus should be on systematically identifying and analyzing further negative factors. In this context, it might be helpful, for instance, to study the impact of good or less good coverages by PVD on the quality of the traffic flow estimates. Moreover, additional effects such as variable speed limits or the like should be considered with regard to future improvements of the proposed method.

Finally, some ideas on their own, namely modelling the relation between macroscopic traffic state variables stochastically and dynamically via Bayesian networks, might prove beneficial also for other applications including online approaches which use the basic concept of the fundamental diagram.

\section{ACKNOWLEDGMENT}

The authors would like to thank VIZ Berlin and Taxi Berlin TZB GmbH for providing the local traffic data and PVD, respectively, which were used in this study.

\section{REFERENCES}

[1] Transportation Research Board, "75 Years of the Fundamental Diagram for Traffic Flow Theory - Greenshields Symposium", Transportation Research Circular, E-C149, 2011.

[2] B. D. Greenshields, "A Study of Traffic Capacity", in Proceedings 14th Annual Meeting, Highway Research Board, 1935, pp. 448-477.

[3] N. Gartner, C. J. Messer, and A. K. Rathi (eds.), Revised Monograph on Traffic Flow Theory, Online ressource: web.tongji.edu.cn/ ryangdy/its/tft/tft.htm, last access: 30/01/2013.

[4] J. S. Drake, J. L. Schofer, and A. D. May jr., "A Statistical Analysis of Speed Density Hypotheses", Highway Research Record, vol. 154, 1967, pp. 53-87.

[5] F. L. Hall, V. F. Hurdle, and J. H. Banks, "Synthesis of Recent Work on the Nature of Speed-Flow and Flow-Occupancy (or Density) Relationships on Freeways", Transportation Research Record, vol. 1365,1992 , pp. 12-18.

[6] M. van Aerde, "A Single Regime Speed-Flow-Density Relationship for Freeways and Arterials", in Proccedings 74th Annual Meeting, Transportation Research Board, Washington, DC, 1995, Paper No. 950802 .

[7] H. Wang, D. Ni, Q. Y. Chen, and J. Li, "Stochastic modeling of the equilibrium speed-density relationship", Journal of Advanced Transportation, vol. 47, 2013, pp. 126-150.

[8] N. Geroliminis, and C. F. Daganzo, "Existence of urban-scale macroscopic fundamental diagrams: Some experimental findings", Transportation Research Part B, vol. 42, 2008, pp. 759-770.

[9] N. Geroliminis, J. Haddad, and M. Ramezani, "Optimal Perimeter Control for Two Urban Regions With Macroscopic Fundamental Diagrams: A Model Predictive Approach", IEEE Transactions on Intelligent Transportation Systems, vol. 14, 2013, pp. 348-359.

[10] R. P. Schäfer, K. U. Thiessenhusen, E. Brockfeld, and P. Wagner, "A traffic information system by means of real-time floating-car data", in Proceedings 9th ITS World Congress, Chicago, IL, 2002.

[11] L. Jie, H. van Zuylen, L. Chunhua, and L. Shoufeng, "Monitoring travel times in an urban network using video, GPS and Bluetooth", Procedia Social and Behavioral Sciences, vol. 20, 2011, pp. 630-637.

[12] C. Nanchawichit, T. Nakatsuji, and H. Suzuki, "Application of Probe Vehicle Data for Real-Time Traffic State Estimation and Short-Term Travel Time Prediction on a Freeway", in Proceedings 82nd Annual Meeting, Transportation Research Board, Washington, DC, 2003.

[13] T. Z. Qiu, X. Y. Lu, A. H. F. Chow, and S. Shladover, "Estimation of Freeway Traffic Density with Loop Detector and Probe Vehicle Data", Transportation Research Record, vol. 2178, 2010, pp. 21-29.

[14] Q. Ou, J. W. C. van Lint, and S. P. Hoogendoorn, "Piecewise Inverse Speed Correction by Using Individual Travel Times", Transportation Research Record, vol. 2049, 2008, pp. 92-102.

[15] Q. Ou, J. W. C. van Lint, and S. P. Hoogendoorn, "An integrated algorithm for fusing travel times, local speed and flow", in Proceedings 13th Conference on Information Fusion, Edinburgh, UK, 2010.

[16] T. Neumann, L. C. Touko Tcheumadjeu, P. L. Böhnke, E. Brockfeld, and X. Bei, "Deriving Traffic Volumes from Probe Vehicle Data using a Fundamental Diagram Approach", in Proceedings 13th World Conference on Transport Research, Rio de Janeiro, Brazil, 2013.

[17] Y. Kim, and H. Keller, "Zur Dynamik zwischen Verkehrszuständen im Fundamentaldiagramm", Straßenverkehrstechnik, vol. 45 (9), 2001, pp. 433-442.

[18] D. Koller, and N. Friedman, Probabilistic Graphical Models - Principles and Techniques, Cambridge, MA: The MIT Press, 2009.

[19] E. Charniak, "Bayesian Networks without Tears", AI Magazine, Winter 1991, pp. 50-63.

[20] Norsys Software Corp., www . nor sys . com, last access: 30/01/2013.

[21] A. Sohr, P. Wagner, and E. Brockfeld, "Floating Car Data based traveltime prediction with Lomb Periodogram", in Proceedings 16th ITS World Congress, Stockholm, Sweden, 2009. 